\title{
Understanding the influence of the electronic structure on the crystal structure of a TTF-PTM radical dyad
}

\author{
Sergi Vela, ${ }^{*}, a$ Manuel Souto, ${ }^{b}$ Imma Ratera,${ }^{*}$, Concepció Rovira,${ }^{b}$ and Jaume Veciana ${ }^{b}$ \\ a) Laboratoire de Chimie Quantique, Université de Strasbourg, 4 rue Blaise Pascal, F-67000 Strasbourg, France. e- \\ mail: \\ b) Institut de Ciència de Materials de Barcelona (ICMAB-CSIC)/CIBER-BBN, Campus de la UAB, 08193, Bellaterra, \\ Spain \\ e-mail address:velallausi@unistra.frandiratera@icmab.es
}

\begin{abstract}
.
The understanding of the crystal structure of organic compounds, and its relationship to their physical properties, have become essential to design new advanced molecular materials. In this context, we present a computational study devoted to rationalize the different crystal packing displayed by two closely-related organic systems based on the TTFPTM dyad (TTF= tetrathiafulvalene, PTM=polychlorotriphenylmethane) with almost the same molecular structure but different electronic one. The radical species (1), with an enhanced electronic donor-acceptor character, exhibits a herringbone packing, whereas the non-radical protonated analogue (2) is organized forming dimers. The stability of the possible polymorphs is analysed in terms of the cohesion energy of the unit cell, intermolecular interactions between pairs and molecular flexibility of the dyad molecules. It is observed that the higher electron delocalization in radical compound $\mathbf{1}$ has a direct influence on the geometry of the molecule, which seems to dictate its preferential crystal structure.
\end{abstract}

\section{Introduction}

Molecular materials can crystallize in multiple structures or polymorphs with different physical properties. Consequently, the modelling of crystallization processes, and polymorph-prediction techniques, have become increasingly important in the quest to improve the structure/function design capabilities. ${ }^{1}$ Progress in this field implies great potential benefits due to their relevance to pharmaceuticals, electronics, and, virtually, all fields of chemical industry working in materials design in the solid state. Most of crystal structure prediction studies are based on the assumption that the crystal structure corresponds to the thermodynamically-favored polymorph. However, the crystal energy landscape is usually more complex, and several polymorphs may be energetically plausible. ${ }^{2}$ Quantum chemistry tools are valuable to gain insight into this particular point, especially when the range of possible polymorphs has been limited by experimental evidence.

In our research, we are focused on the design of materials based on organic molecules formed by an electron donor (D) and an electron acceptor (A) units linked by a conjugated bridge. These are very interesting materials due to their potential application in organic light-emitting devices, non-linear optics, molecular electronics and photonics. ${ }^{3-4}$ The physical properties of such advanced materials are directly related to their molecular packing in the solid state. ${ }^{5}$ In this context, we have recently reported the radical and the non-radical analogue species based on the electron-donor tetrathiafulvalene (TTF) unit connected, through a $\Pi$-conjugated phenyl-pyrrole-vinylene bridge, to the electronacceptor polychlorotriphenylmethane (PTM) radical (1), or to the corresponding $\alpha$ HPTM non-radical derivative (2). Compounds $\mathbf{1}$ and $\mathbf{2}$ exhibit a similar molecular structure but, surprisingly, they present a different crystalline packing. ${ }^{6}$ The radical dyad 1 crystallizes in the monoclinic system (space group $P 2_{1}$ ) forming a herringbone $(h b)$ packing with 
segregated $\mathrm{D}$ and $\mathrm{A}$ units and short $\mathrm{S} \cdots \mathrm{S}$ interactions. In turn, compound $\mathbf{2}$ crystallizes in the triclinic system $(P \overline{1}$ space group), showing face-to-face TTF-dimers (dim) (see Fig. 1$)^{6}$
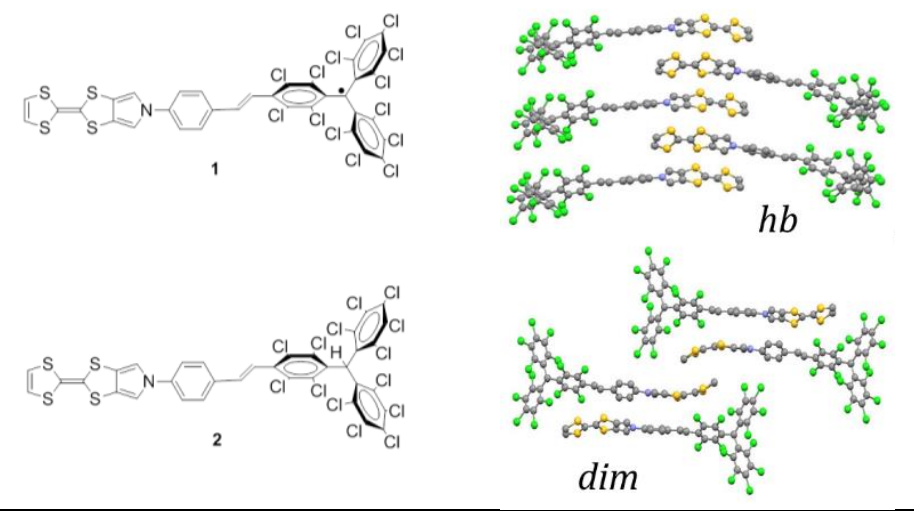

Fig. 1. Molecular structures of radical dyad 1 and non-radical dyad 2 and schematic representation of their crystal arrangements, herringbone $(\mathrm{hb})$ and dimerized (dim), respectively.

TTF-based dyads are of prime interest due to their potential application in electronic and optoelectronic devices. ${ }^{7}$ Among them, those exhibiting a molecular packing with a complete segregation of $\mathrm{D}$ and $\mathrm{A}$ fragments are even more interesting. The reason is that, in order to obtain conducting materials, it would be necessary to achieve a certain degree of charge transfer between the D and A units to promote self-doping as it occurs in most of organic charge transfer (CT) complexes. This conducting behavior is more often accomplished in materials displaying segregated stacks, in which the D-A pairs are better overlapped. ${ }^{8-9}$ For the opposite reason, a polymorph with alternated stacks will mostoften be insulator. ${ }^{10}$ In other words, the conducting properties of charge transfer salts based on two components are highly susceptible to the presence of polymorphism. In our case, the herringbone packing of radical dyad $\mathbf{1}$, showing segregated stacks of TTF and PTM units, is optimal for exhibiting electrical conductivity. Indeed, it has been recently proven that the conductivity of $\mathbf{1}$ occurs under high pressure, once the overlap between molecules is sufficiently large and there is a certain degree or charge reorganization. ${ }^{10}$ On the contrary, the dimerized packing adopted by compound 2 does not show such potential since the TTF units are not properly organized. For these reasons, it is important to understand the origin of the different supramolecular assemblies displayed by $\mathbf{1}$ and $\mathbf{2}$. Is it due to a change in the electronic structure of the PTM moiety (open vs. closed-shell)? Are the two polymorphs plausible for the two compounds? The main goal of the manuscript is to provide an answer to these questions, whose rationalization is of interest to the prediction of the crystal structures and physical properties of D-A systems and, more specially, of those based on TTF-PTM dyads.

In this article we report a computational study devoted to rationalize the occurrence of the $h b$ and $\operatorname{dim}$ crystal packings for compounds $\mathbf{1}$ and $\mathbf{2}$. Based on the crystallographic structures reported experimentally ( $h b$ and $\operatorname{dim}$ for $\mathbf{1}$ and $\mathbf{2}$, respectively), the hypothetical structures of 1-dim and 2- $h b$ have been computed. The absolute energies of the four resulting structures have been analyzed, together with the structure and energy of the constituent molecular units. In doing so, we can ascribe the structural changes observed between the individual units of $\mathbf{1}$ and $\mathbf{2}$ to either the modification of their electronic structure, or to the effect of the crystal-packing arrangement. Moreover, we have also computed the cohesion energy of the lattices, and the intermolecular interaction energies between selected pairs. Altogether, this work allows us to quantify the structural characteristics and stability of compounds $\mathbf{1}$ and $\mathbf{2}$ in the two possible polymorphs and, more important, to connect the results with the molecular structure of the units, and the supramolecular interactions between them in order to understand the influence of the electronic structure on the crystal structure. 


\section{Results and Discussion}

1. Polymorph Stability. In order to perform a meaningful energetic comparison between the different structures of both compounds, it is first necessary to obtain the minimum-energy structure of all possible polymorphs. That is, those representing the packing of the two studied compounds $(\mathbf{1}$ and $\mathbf{2})$ in either a herringbone $(h b)$ or a dimerized (dim) configuration. We have labelled these cells as 1-dim, 1- $h b, \mathbf{2}-\operatorname{dim}$ and 2-hb, and their analysis will allow us to rationalize to what extent the changes in the supramolecular architectures are originated in the electronic structure of the constituting units. With this aim in mind, we have performed variable-cell geometry optimizations of 1- $h b, \mathbf{1}$-dim, 2- $h b$ and 2-dim, whose initial geometry has been taken (for 1- $h b$ and 2-dim) or adapted (for 1-dim and 2-hb) from the reported unit cells by the addition (or subtraction) of the hydrogen atom of the PTM moiety. ${ }^{11}$ Along the text, we will refer to the predicted structures of 1-dim and 2- $h b$ as polymorphs. We do so for simplicity in the discussion, but it must be clear that these structures have not been reported experimentally. The stability of the resulting unit cells has been collected in Table 1, and shows an overestabilization of the dimerized arrangement by ca. $4.5 \mathrm{~kJ} / \mathrm{mol}$ (per molecule) with respect to the herringbone one, for both compounds. Such small energy differences are common in computational studies of polymorphism, especially for non-conformational polymorphs as in the present case. ${ }^{12-13}$

Table 1. Absolute energy (in Ry) and relative energy difference (in $\mathrm{kJ} / \mathrm{mol}$ ) of the unit cells, which include 4 monomers of either $\mathbf{1}$ or $\mathbf{2}$, arranged in either a herringbone $(h b)$ or a dimerized $(\mathrm{dim})$ pattern. $\Delta E$ is given per molecule.

\begin{tabular}{ccc}
\hline & $\mathbf{1}$ & $\mathbf{2}$ \\
\hline$h b(\mathrm{Ry})$ & -3841.905 & -3846.866 \\
$\operatorname{dim}(\mathrm{Ry})$ & -3841.919 & -3846.880 \\
\hline$\Delta E(\mathrm{~kJ} / \mathrm{mol})$ & 4.73 & 4.44 \\
\hline
\end{tabular}

The stability of crystal structures can be roughly separated into two contributions: (i) the stability of the molecular geometries of the monomers $\left(E_{g e o}\right)$ and the (ii) amount of intermolecular interactions between them. The former can be evaluated by calculating the energy of the isolated molecules as in eq. 1, whereas the latter can be evaluated by means of the cohesion energy ( $E_{c o h}$, eq. 2), where $E_{\text {mon, } i}$ is the energy of monomer $i$ at its geometry in the optimized crystalline unit cell, whose energy is $E_{\text {cell }}$.

$$
\begin{aligned}
E_{\text {geo }} & =\sum_{i=1,4} E_{\text {mon, } i} \\
E_{\text {coh }} & =E_{\text {cell }}-E_{\text {geo }}
\end{aligned}
$$

We have analyzed the four crystal structures using this scheme (see Table 2). First of all, it must be noticed that the super-cells employed to compute the minima of polymorphs 1-dim, 1- $h b, \mathbf{2}$ - $\operatorname{dim}$ and 2- $h b$ contain four monomers (A$\mathrm{D}$ ). The absolute energies of monomers $\mathrm{A}$ and $\mathrm{B}$ are notably different than those of monomers $\mathrm{C}$ and $\mathrm{D}$ (see $\Delta E_{\text {mon }}$ in Table 2, and Fig. 2 for labelling). This is due to the presence of two types of crystallographic asymmetric units in the crystal structures, as resolved experimentally. Overall, these results show that the stability of the herringbone polymorphs is favored, with respect to the dimerized polymorphs, both in $\mathbf{1}$ and $\mathbf{2}$, by the larger stability of the individual molecules (see $\Delta E_{g e o}$ in Table 2). However, the dimerized polymorphs are more stable than the herringbone due to the fact that they are capable to maximize the cohesion energy thanks to larger attractive intermolecular interactions (see $\Delta E_{c o h}$ in Table 2). These two points will be analysed in detail in the following sections.

Table 2. Absolute energies (in Ry) of the four molecules comprising the unit cells studied $\left(E_{m o n, i}\right)$, calculated in the gas phase and using their optimized geometry in the crystal. The unit cells have two types of molecules (A\&B and C\&D, see main text and Figure 2). The energy difference between each type of molecules is $\Delta E_{\text {mon }} . E_{g e o}$ is calculated from eq. $1 . \Delta E_{g e o}$ is the difference in $E_{g e o}$ between $h b$ and $\operatorname{dim}$ crystals of a given compound. $E_{c o h}$ is calculated from eq. 2 using the unit-cell energies given in Table 1. $\Delta E_{m o n}, \Delta E_{g e o}, \Delta E_{c o h}$ and $E_{c o h}$ are given in $\mathrm{kJ} / \mathrm{mol}$ (per molecule), whereas $E_{m o n, 1-2}$ and $E_{g e o}$ are given in Ry.

\begin{tabular}{ccccc} 
& $\mathbf{1 - d i m}$ & $\mathbf{1 -}-\mathrm{hb}$ & $\mathbf{2 - d i m}$ & $\mathbf{2 - h b}$ \\
\hline$E_{\text {mon }, A-B}$ & -960.170 & -960.184 & -961.408 & -961.422
\end{tabular}




\begin{tabular}{|c|c|c|c|c|}
\hline$E_{\text {mon }, C-D}$ & -960.180 & -960.187 & -961.422 & -961.426 \\
\hline$\Delta E_{\text {mon }}$ & 12.7 & 4.0 & 18.0 & 4.9 \\
\hline$E_{g e o}$ & -3840.701 & -3840.743 & -3845.661 & -3845.697 \\
\hline$\Delta E_{\text {geo }}$ & \multicolumn{2}{|c|}{13.9} & \multicolumn{2}{|c|}{11.9} \\
\hline$E_{c o h}$ & -399.5 & -380.9 & -399.7 & -383.3 \\
\hline$\Delta E_{c o h}$ & \multicolumn{2}{|c|}{-18.6} & \multicolumn{2}{|c|}{-16.4} \\
\hline
\end{tabular}

2. Analysis of crystal \& isolated structures. To understand the absolute energy of the monomers of $\mathbf{1}$ and $\mathbf{2}$ in each polymorph, we have first analysed the structural characterization of the molecules in the computed crystals. We have chosen a comprehensive set of distances and torsion angles that cover both the internal structural characteristics of the different molecular subunits (i.e. TTF, vinylene, PTM...) and their relative disposition. As previously mentioned, there are two asymmetric units in the cells (monomers $\mathrm{A}$ and $\mathrm{B} v s . \mathrm{C}$ and $\mathrm{D}$ ). This means that such variables may be different for each type of them. We have seen that this is not the case when analyzing the distances, but it is certainly true with torsion angles. Therefore, some of the variables analyzed in Table 3 display two values, one for each type of molecule in the unit cell. Thanks to this analysis, we can ascribe all structural differences between $\mathbf{1}$ and $\mathbf{2}$ to either the electronic structure or the crystal-packing. For instance, it is observed that the TTF unit is slightly more planar (i.e. smaller angle) in the radical derivative $\mathbf{1}$ than in 2, and also in the Herringbone than in the Dimerized polymorphs (see Fig. 3). The first point can be explained by the loss of electron density (higher delocalization) in the TTF unit of radical compound 1 due to the much higher electron-acceptor character of PTM radical (the neutral TTF exhibits a boat conformation whereas the oxidized $\mathrm{TTF}^{+\cdot}$ is planar ${ }^{14-15}$ ). The second point, in turn, can be ascribed to the larger steric hindrance between the bulky PTM and the TTF units in the dimerized arrangement. Regarding the bridging units connecting the $\mathrm{D}$ and A moieties, no major differences are observed in the vinylene bridge region, in contrast to what is observed for the Phenyl-Pyrrole twist, which displays significant changes depending on the compound, the polymorph, and the type of monomer (from $2.0^{\circ}$ to $38.1^{\circ}$ ) and, thus, seems to be the most flexible piece of $\mathbf{1}$ and $\mathbf{2}$. Finally, it is worth mentioning that the ipso- $C$ (C20-C23, $\mathrm{C} 23-\mathrm{C} 24$ and $\mathrm{C} 23-\mathrm{C} 30)$ and $\mathrm{C}-\mathrm{Cl}$ average distances are always shorter for $\mathbf{1}$ in agreement with the $\mathrm{sp}^{2}$ configuration of the radical and the partial delocalization of the unpaired electron into the aromatic rings. ${ }^{6}$, 15
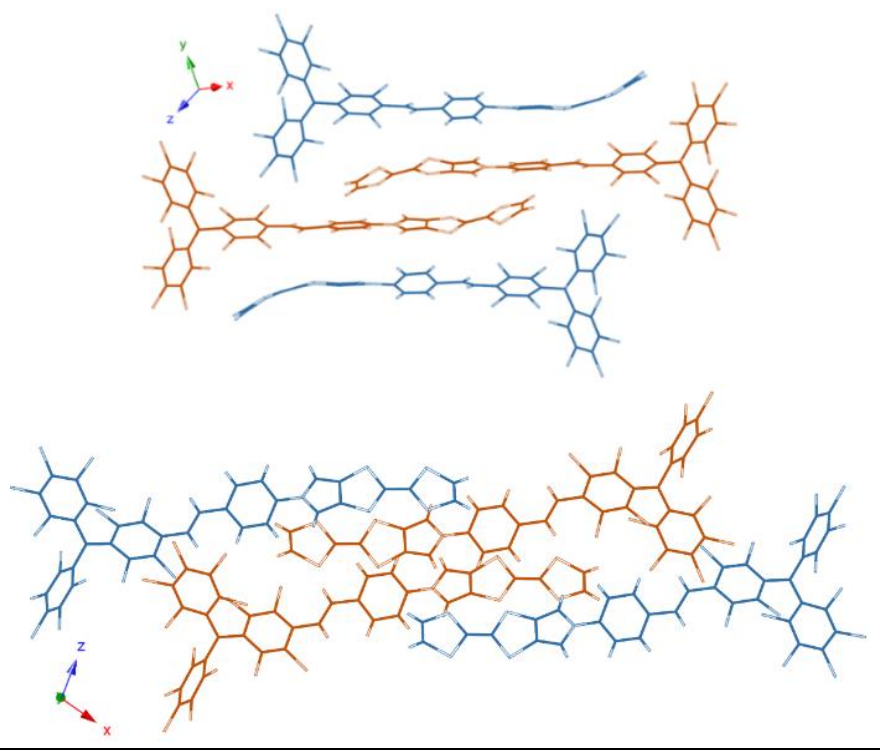

Fig. 2. Representation of the Dimerized (top) and Herringbone (below) optimized Unit cells of 1 showing the different types of monomers: $\mathrm{A}$ and $\mathrm{B}$ in blue, $\mathrm{C}$ and $\mathrm{D}$ in red.

The solid-state optimized geometries are further compared to those obtained from solvent-phase calculations (isolated: 1-iso, 2-iso) (Fig. 4). Such comparison allows us to identify to what extent the molecules are distorted in the unit cells 
with respect to their minima in the solvent used in the crystallization, a 1:1 mixture of di-chloromethane and hexane. ${ }^{5}$ The most important changes are observed again in the TTF units and the Phenyl-Pyrrole twist angles. First, regarding the co-planarity of the TTF units, it can be clearly seen that it is largely determined by the electronic structure of the molecule $\left(12.3^{\circ}\right.$ in 1-iso and $37.9^{\circ}$ in 2-iso). This fact also indicates that TTF units are, indeed, largely distorted in both polymorphs of $\mathbf{1}$ (especially in 1- $\mathrm{dim}$ ) from their optimum value of $12.3^{\circ}$, whereas molecules of $\mathbf{2}$ display similar TTF co-planarity angles in the two polymorphs and in solvent-phase. The relative differences between polymorphs of 1 have been rationalized above. Second, important differences are also observed in the Phenyl-Pyrrole twist, which is much less distorted in the minima of the experimentally-obtained polymorphs (1-hb and 2-dim) than in 1-dim and 2$h b$. It must be mentioned that this distortion is even more significant for one type of monomers in 1 -dim $\left(\mathrm{ca} .23 .6^{\circ}=\right.$ $25.6-2.0$, see Table 3). We believe these distortions could play a key role in determining the polymorph that is obtained experimentally, as we will discuss in the following. In turn, the vinylene region does not display any major distortion. Finally, we would like to mention that the solvent-phase structures (1-iso, 2-iso) are extremely close to the gas-phase structures (ie. without solvent), which suggests that the solvent does not have any major influence.

Table 3. Selected intramolecular distances, angles and torsion angles of compounds $\mathbf{1}$ and $\mathbf{2}$ obtained from the dimerized (dim) and herringbone $(h b)$ arrangements and from the solvent-phase calculations (iso).

\begin{tabular}{|c|c|c|c|c|c|c|c|}
\hline Unit & Distances $(\AA)$ & 1-dim & $1-h b$ & 1-iso & 2-dim & $2-h b$ & 2-iso \\
\hline Phenyl-Pyrrole & $d(C-N)$ & 1.41 & 1.41 & 1.41 & 1.41 & 1.41 & 1.41 \\
\hline TTF & $\bar{d}(C-S)^{\mathrm{a}}$ & 1.76 & 1.76 & 1.77 & 1.76 & 1.76 & 1.78 \\
\hline TTF & $\bar{d}(C=C)^{\mathrm{b}}$ & 1.38 & 1.38 & 1.38 & 1.38 & 1.37 & 1.38 \\
\hline PTM & $\bar{d}(C-C l)$ & 3.90 & 3.91 & 3.83 & 4.07 & 4.17 & 4.10 \\
\hline PTM & $\bar{d}(\text { ipso }-C)^{\mathrm{c}}$ & 1.46 & 1.46 & 1.46 & 1.53 & 1.53 & 1.53 \\
\hline Angles $\left(^{\circ}\right)$ & Monomer & 1-dim & 1- $h b$ & 1-iso & 2-dim & $2-h b$ & 2-iso \\
\hline TTF co-planarity ${ }^{\mathrm{d}}$ & $\begin{array}{l}1 \& 2 \\
3 \& 4\end{array}$ & $\begin{array}{l}31.6 \\
29.2\end{array}$ & $\begin{array}{l}21.9 \\
21.1\end{array}$ & 12.3 & $\begin{array}{l}34.8 \\
29.9\end{array}$ & $\begin{array}{l}23.6 \\
28.2\end{array}$ & 37.9 \\
\hline Phenyl-Pyrrole twist ${ }^{\mathrm{e}}$ & $\begin{array}{l}1 \& 2 \\
3 \& 4\end{array}$ & $\begin{array}{c}2.0 \\
14.5\end{array}$ & $\begin{array}{l}35.5 \\
28.6\end{array}$ & 27.9 & $\begin{array}{l}21.7 \\
23.2\end{array}$ & $\begin{array}{l}38.1 \\
28.1\end{array}$ & 28.8 \\
\hline Vinylene bridge $\mathrm{f}^{\mathrm{f}}$ & $\begin{array}{l}1 \& 2 \\
3 \& 4\end{array}$ & $\begin{array}{l}176.1 \\
174.3\end{array}$ & $\begin{array}{l}171.6 \\
177.7\end{array}$ & 179.8 & $\begin{array}{l}172.7 \\
170.9\end{array}$ & $\begin{array}{l}171.5 \\
178.3\end{array}$ & 179.2 \\
\hline
\end{tabular}

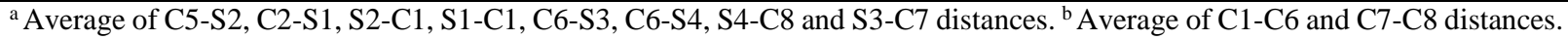
${ }^{\mathrm{c}}$ Average of $\mathrm{C} 20-\mathrm{C} 23, \mathrm{C} 23-\mathrm{C} 24$ and $\mathrm{C} 23-\mathrm{C} 30$ distances. ${ }^{\mathrm{d}}$ Angle between the planes formed by S3-C7-C8-S4 and S2-C5-C2S1. ${ }^{\mathrm{e}}$ Average of torsion angles of C14-C9-N1-C4 and C10-C9-N1-C3. ${ }^{\mathrm{f}}$ Torsion angle of C12-C15-C16-C17. See Fig. 3 for labels.

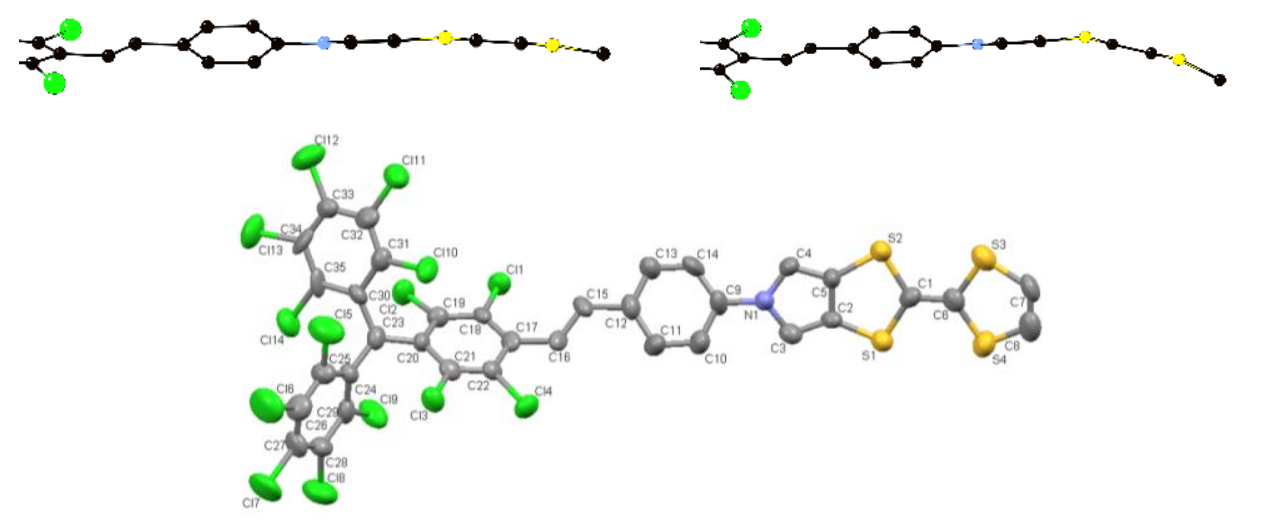

Fig. 3. TTF region of the Solvent-phase optimized geometries of (top-left) radical compound 1 and (top-right) compound 2, showing the different planarity of the TTF moiety for the two dyads. (below) Molecular structure of radical dyad $\mathbf{1}$ with atomic labels, as used in Table 3 . The grey, green, blue and yellow ellipsoids represent $\mathrm{C}, \mathrm{Cl}, \mathrm{N}$ and $\mathrm{S}$ atoms, respectively. 
3. Analysis of interaction energies. Once we have analyzed the structural properties of the molecules in the studied polymorphs and in gas-phase, it is now worth to analyze the strength of the interaction energy between pairs of molecules $\left(E_{\text {int }}\right)$ in the crystals. To do so, we have extracted the nuclear coordinates of a set of selected dimers from the optimized cells of the four polymorphs. Then, we have evaluated the strength of $E_{\text {int }}$ and the contribution of the van der Waals contacts (see Computational Details section and Table 4). Three different pairs. have been analyzed for the dim-type cells and four for the $h b$-type ( $1^{\text {st }}$ neighbors closer than ca. $12 \AA$, see Fig. 4$)$. For the dim-type cells, the "short" pair is the one with larger attractive intermolecular interactions (ca. $60 \mathrm{~kJ} / \mathrm{mol}$ ), whereas the "lateral" and "slipped" pairs display weaker (ca. $33.2 \mathrm{~kJ} / \mathrm{mol}$ ) and almost-negligible (ca. $4 \mathrm{~kJ} / \mathrm{mol}$ ) interactions, respectively. These results seem to follow one clear trend, the more overlap, the larger the interaction. This was certainly expected, given that the van der Waals contacts are responsible for $60-85 \%$ of the strength of these interactions (Table 4). Regarding the $h b$-type cells, the "lateral" pair is the one displaying larger attractive intermolecular interactions $(c a .48 \mathrm{~kJ} / \mathrm{mol})$,

Table 4. Intermolecular Interaction Energies $\left(E_{\text {int }}\right.$, in $\mathrm{kJ} / \mathrm{mol}$ ) for selected pairs of molecules (dimers) in the solid-state minima of the dim and $h b$ polymorphs. It is also shown the contribution of the van der Waals contacts. The dimers are displayed in Fig. 2 and are also identified here by the distance between $\mathrm{N}$ atoms (in $\AA$ ). Highlighted in grey the results for the experimentallyobtained polymorphs.

\begin{tabular}{ccccccc}
\hline $\boldsymbol{d i m}$ & $\mathrm{d}(\mathrm{N}-\mathrm{N})$ & $E_{\text {int }}$ & $\mathrm{vdW}$ & $\mathrm{d}(\mathrm{N}-\mathrm{N})$ & $E_{\text {int }}$ & $\mathrm{vdW}$ \\
\hline Short & 3.5 & -59.4 & $85 \%$ & 4.1 & -62.1 & $87 \%$ \\
Lateral & 7.3 & -33.2 & $88 \%$ & 7.4 & -33.6 & $90 \%$ \\
Slipped & 12.2 & -0.4 & $58 \%$ & 9.9 & -6.8 & $59 \%$ \\
\hline $\boldsymbol{h b}$ & $\mathrm{d}(\mathrm{N}-\mathrm{N})$ & $E_{\text {int }}$ & $\mathrm{vdW}$ & $\mathrm{d}(\mathrm{N}-\mathrm{N})$ & $E_{\text {int }}$ & $\mathrm{vdW}$ \\
\hline Short & 4.8 & -23.9 & $72 \%$ & 4.9 & -28.7 & $67 \%$ \\
Lateral & 8.7 & -46.6 & $76 \%$ & 8.7 & -49.5 & $77 \%$ \\
Diagonal \#1 & 9.0 & -20.2 & $67 \%$ & 8.9 & -23.7 & $72 \%$ \\
Diagonal \#2 & 10.8 & -7.0 & $60 \%$ & 10.8 & -6.3 & $66 \%$ \\
\hline
\end{tabular}

followed by the "short" and "diagonal\#1" pairs ( $c a .25 \mathrm{~kJ} / \mathrm{mol}$ ) and, finally, the "diagonal\#2" pair (ca. $7 \mathrm{~kJ} / \mathrm{mol})$. This analysis is relevant as it indicates that each type of crystal packing ( $h b$ and dim) is governed by a different dominant pair, in which the two molecules arrange in either an anti-parallel fashion (the "short" pair in the dimerized packing) or perpendicularly (the "lateral" pair in the herringbone packing).

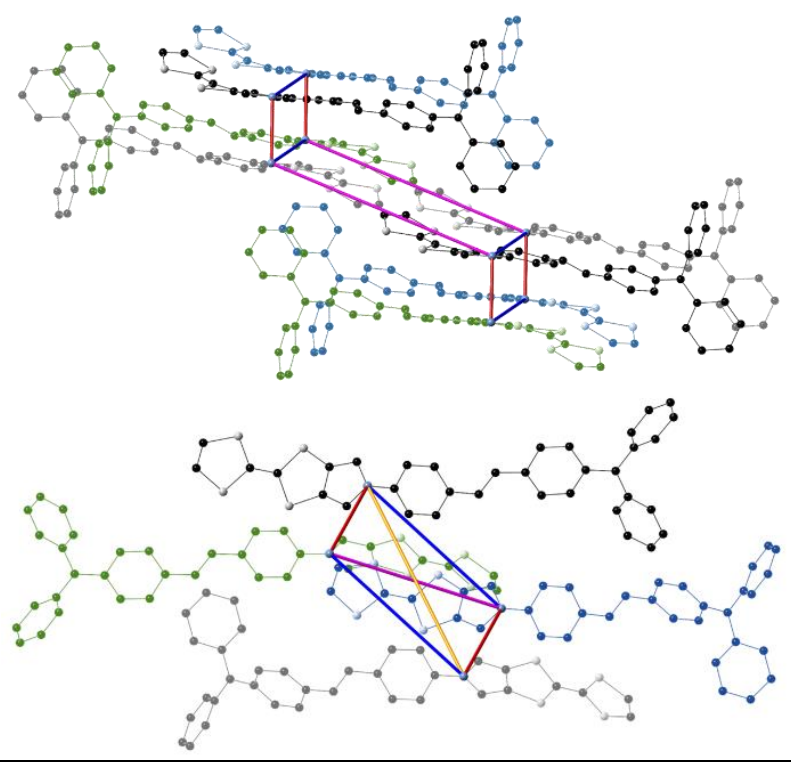

Fig. 4. Scheme of the different pairs selected for the analysis of $E_{\text {int }}$ in the Dimerized (dim, top) and Herringbone ( $h b$, below) polymorphs (see Table 
4). Color code for pairs: Red (Short) Blue (Lateral) Purple (Slipped for 1 and Diagonal \#1 for 2) Orange (Diagonal \#2). For clarity, $\mathrm{Cl}$ and $\mathrm{H}$ atoms have been omitted and molecules have been coloured.

It is also worth to mention that the intermolecular distances suffer notable changes in the dimerized cells for $\mathbf{1}$ and $\mathbf{2}$. In particular, the "short" pair in dimerized cells is much affected depending on the compound. The $\mathrm{N} \cdots \mathrm{N}$ distance increases from $3.5 \AA$ to $4.1 \AA$ and the distance between molecular planes goes from $3.5 \AA$ to $3.8 \AA$. In parallel, the TTF plane becomes more distorted (i.e. from 8.13 to $10.31^{\circ}$ ), partially as a result of the structural (and electronic) changes undergone by the PTM moiety (from $\mathrm{sp}^{2}$ to $\mathrm{sp}^{3} \mathrm{C}$ ). These changes in the "short" pair also imply a modification of the intermolecular distance between the other pairs. In particular, the $\mathrm{N} \cdots \mathrm{N}$ distance for the "slipped" pair is significantly modified. Such important changes in the unit cell are not observed in the Herringbone arrangement (1- $h b$ and 2- $h b$ ), which seems to be more robust, ie. less prone to changes in the relative distance between units. This might also be related to the larger cohesion energies $\left(E_{c o h}\right)$ observed for the dimerized cells: their flexibility allows the molecules to maximize their intermolecular interactions $\left(E_{\text {int }}\right)$, at the expense of suffering a larger energy penalty for the molecular deformation (see Table 2). Instead, in the herringbone cells there is no such possibility, so the molecules maximize their internal stability at the expense of a less-efficient $E_{c o h}$. This can also be seen in the unit-cell volume and density- changes between the computed and experimentally-obtained polymorphs (see Table 5). The $h b$-type unitcells barely display any sort of thermal lattice compression upon cooling (indeed, there is even a small expansion), whereas the dim-type cells display notably smaller unit-cell parameters and, thus, larger densities, as temperature is "decreased" (experimental at $300 \mathrm{~K} v s$. optimized at formally $0 \mathrm{~K}$ ). We believe this might be also an indication of the larger "robustness" of the $h b$ unit-cells.

Table 5. Unit cell parameters and characteristics of the four explored polymorphs of $\mathbf{1}$ and $\mathbf{2}$ and the experimentally-reported crystals. The optimized (opt) supercells correspond, formally, to the $0 \mathrm{~K}$ structure of the crystals.

\begin{tabular}{ccccccc}
\hline & $\mathbf{1 -} h b$ & 1-dim & $\mathbf{1 -} h b$ & $\mathbf{2 -} h b$ & 2-dim & 2-dim \\
\hline Type & opt & opt & exp & opt & opt & exp \\
$\mathrm{T}(\mathrm{K})$ & 0 & 0 & 300 & 0 & 0 & 300 \\
\hline$a(\AA)$ & 17.49 & 7.32 & 17.41 & 17.35 & 7.36 & 8.78 \\
$b(\AA)$ & 8.07 & 21.50 & 7.95 & 8.19 & 22.30 & 22.27 \\
$c(\AA)$ & 29.69 & 28.02 & 30.17 & 29.57 & 27.44 & 25.44 \\
\hline$\alpha\left(^{\circ}\right)$ & 90.0 & 87.4 & 90.0 & 90.1 & 94.2 & 95.98 \\
$\beta\left({ }^{\circ}\right)$ & 102.9 & 92.8 & 103.6 & 102.0 & 88.1 & 94.71 \\
$\gamma\left({ }^{\circ}\right)$ & 90.0 & 103.4 & 90.0 & 90.0 & 107.9 & 112.22 \\
\hline $\mathrm{V}\left(\AA^{3}\right)$ & 4080.5 & 4283.7 & 4061.0 & 4105.9 & 4277.9 & 4636.0 \\
\hline $\mathrm{d}\left(\mathrm{g} / \mathrm{cm}^{3}\right)$ & 1.74 & 1.66 & 1.75 & 1.73 & 1.66 & 1.53 \\
\hline
\end{tabular}

4. Possible rationalization of the polymorphism of 1 and 2. Our calculations suggest that the stability of the $h b$ and dim polymorphs is very similar for both $\mathbf{1}$ and $\mathbf{2}$. Therefore, we cannot rationalize their crystallization in different polymorphs based on sound thermodynamic considerations. If any further rationalization is possible, we should turn our attention to any potential differential factor driving the kinetics of their nucleation-and-growth process. The computational rationalization of this scenario is much more complex, if not impossible. However, the structural analysis of the individual units in the polymorph minima, prompts us to suggest a possible explanation for the different type of crystal packing observed in compounds $\mathbf{1}$ and $\mathbf{2}$. As we have just mentioned, the crystallization of the two compounds might to be governed by kinetics. In other words, by a difference in the energy barriers that connect the initial structure (ie. the solvent-phase minima) with the final structures (ie. in the crystal) in each of the two possible polymorphs. We propose that this energy barrier is related to the degree of geometrical distortion of the monomers. Intuitively, larger distortions must be associated with larger barriers and, thus, the kinetics of the process might favour the formation of the polymorphs in which the molecules display a smaller geometrical distortion. This seems to be the case of compounds $\mathbf{1}$ and $\mathbf{2}$, in which the differential factor is a change in the electronic structure of one component of the TTF-PTM dyad (PTM radical vs. non-radical). This small change has an influence on the geometry of the other 
component (TTF moiety), with the side-effect of an additional distortion of the bridging unit (Phenyl-Pyrrole twist). As a result, compounds $\mathbf{1}$ and $\mathbf{2}$ present a notably-different minimum in solvent, especially regarding the co-planarity of the TTF moiety (1-iso vs. 2-iso, Table 3). The TTF is much more planar in $\mathbf{1}$ than in $\mathbf{2}$ (see Figure 4), and planar TTF moieties are better accommodated in the $h b$ packing than in the dim packing, since, in the latter, they have a larger steric hindrance with the bulky PTM (see structural analysis). This is one possible reason of why $\mathbf{1}$ would prefer the $h b$ packing rather than the dim one. In turn, the polymorph found for compound $\mathbf{2}$ seems easier to rationalize. It is both the most stable thermodynamically and the one in which the molecules present smaller distortions with respect to the solvent-phase structure. Finally, it may be worth mentioning as a side-note that, during the preparation of the two studied compounds for our previous experimental work, ${ }^{6}$ the crystallization of $\mathbf{1}$ was more 'difficult' than that of $\mathbf{2}$. This agrees with the analysis above, and with the fact that the degree of distortion is, indeed, larger for $\mathbf{1}$ than for $\mathbf{2}$.

\section{Computational Details}

The solid state calculations have been performed using the Quantum Espresso package (QE) ${ }^{16}$ the PBE functional within the spin unrestricted formalism, the D2 correction of Grimme, ${ }^{17}$ Vanderbilt pseudopotentials and a $\Gamma$-point sampling of the Brillouin zone. It must be mentioned that DFT-functionals incorporating dispersion correction has been a common method for studying polymorphism in molecular crystals. ${ }^{1}{ }^{18-24}$ The minimum energy structure of the different polymorphs has been obtained by performing successive variable-cell geometry relaxations, in which the lattice parameters as well as the atomic positions are optimized simultaneously until the atomic forces are smaller than $1.0 \mathrm{E}^{-5}$ atomic units. We have chosen to compare optimized structures (instead of the reported crystals) for two reasons. Most importantly, because the two hypothetical polymorphs would not be realistic without an optimization procedure. Moreover, this is also positive as it ensures that temperature (ie. entropy) effects do not play any role (as expected) in the structural comparison between polymorphs of the same compound. In the geometry-optimization calculations in the crystalline phase, the number of plane waves has been kept constant at a kinetic energy cut-off of 70 Ry throughout the variable-cell relaxations. An antiferromagnetic spin state has been assumed for the radical species (1) through the definition of an appropriate initial guess that is maintained along the optimization. The energy of the monomers to evaluate the cohesion energy has been computed by performing a single-point calculation of the selected molecules, each in their optimized geometry within the optimized unit cell (ie. no further optimization). All solvent-phase optimizations, and the evaluation of the intermolecular interactions, have been carried our using the PBE functional, a $6-311+\mathrm{G}(\mathrm{d})$ basis set, and the D2 correction of Grimme ${ }^{17}$ as implemented in Gaussian 09. ${ }^{25}$ For the former, the PCM model has been employed to simulate the solvent, a 1:1 mixture of hexane and di-chloromethane. Only the transconformation has been explored in the study of the isolated structures, since it is the only conformation present in the crystal. The cis-conformation has been found to be $c a .12 \mathrm{~kJ} / \mathrm{mol}$ higher in energy in the highly related PTM-TTFPTM triad. ${ }^{26}$

\section{Conclusions}

We have performed a computational analysis of the relative stability of the $\operatorname{dim}$ and $h b$ polymorphs of compounds $\mathbf{1}$ and 2, which reveals that the energy difference between them is quite small in both cases (ca. $4.5 \mathrm{~kJ} / \mathrm{mol}$, see Table 1), favoring the $\operatorname{dim}$ polymorph. The analysis of molecular \& lattice stabilities shows that the constituting units are slightly more stable in the $h b$ than in the dim polymorphs, both in the case of $\mathbf{1}$ and $\mathbf{2}$ (see $\Delta E_{g e o}$ in Table 2). However, the $\operatorname{dim}$ polymorphs are globally more stable due to the fact that they are capable to maximize the intermolecular interaction energies $\left(E_{\text {int }}\right)$, thus increasing the cohesion energy of the lattice. A deeper study on this point quantified the magnitude of those interactions for the most relevant pairs of molecules in the crystal packing, and showed that the $h b$ and $\operatorname{dim}$ crystal packings have a different dominant pair (so-called lateral vs. short pairs). The relative stability of the polymorphs, which slightly favours the $\operatorname{dim}$ crystals, is in apparent disagreement with the fact that the experimentallyobserved polymorph of $\mathbf{1}$ displays the $h b$ packing. Considering the error associated to our calculations, and based on an energetic criterion, we can only conclude that the two polymorphs are thermodynamically plausible. However, our 
structural analysis indicates that the individual molecules of 1 and 2 present a notably-different minima in solventphase, originated in their different electronic structure. This observation prompted us to propose an explanation to understand their polymorphism, based on the degree of distortion that the individual molecules undergo from their minima in the solvent-phase to their minima in the crystal.

\section{Supporting Information}

S1-Cartesian coordinates of the optimized polymorph structures.

\section{Acknowledgements}

This work was supported by the DGI grant (CTQ2013-40480-R), the Networking Research Center on Bioengineering, Biomaterials, and Nanomedicine (CIBER-BBN), the EU ITN iSwitch 642196, the NANO2FUN Project No. 607721 and the Generalitat de Catalunya (grant 2014-SGR-17). ICMAB acknowledges support from the Spanish Ministry of Economy and Competitiveness, through the "Severo Ochoa" Programme for Centres of Excellence in R\&D (SEV2015-0496). S.V. is thankful to the LabEx-Chemistry of Complex Systems for a post-doctoral grant (ANR-10-LABX0026CSC) and to the regional High-Performance Computing (HPC) center in Strasbourg for computational resources. M.S. is enrolled in the Material Science Ph.D. program of UAB and is grateful to MEC for a FPU predoctoral grant.

\section{References}

1.Beran, G. J. O., Modeling Polymorphic Molecular Crystals with Electronic Structure Theory. Chem. Rev. (Washington, DC, U. S.) 2016, $116(9), 5567-5613$.

2.Price, S. L., Predicting crystal structures of organic compounds. Chem. Soc. Rev. 2014, 43 (7), 2098-2111.

3.Ratera, I.; Veciana, J., Playing with organic radicals as building blocks for functional molecular materials. Chem. Soc. Rev. 2012, 41 (1), 303-349.

4.Chu, C. W.; Ouyang, J.; Tseng, J. H.; Yang, Y., Organic Donor-Acceptor System Exhibiting Electrical Bistability for Use in Memory Devices. Adv. Mater. (Weinheim, Ger.) 2005, 17 (11), 1440-1443.

5.Bernstein, J., Crystal growth, polymorphism and structure-property relationships in organic crystals. J. Phys. D: Appl. Phys. 1993, 26 (8B), B66.

6.Souto, M.; Solano, M. V.; Jensen, M.; Bendixen, D.; Delchiaro, F.; Girlando, A.; Painelli, A.; Jeppesen, J. O.; Rovira, C.; Ratera, I.; Veciana, J., Self-Assembled Architectures with Segregated Donor and Acceptor Units of a Dyad Based on a Monopyrrolo-Annulated TTF-PTM Radical. Chem. Eur, J. 2015, 21 (24), 8816-8825.

7.Bendikov, M.; Wudl, F.; Perepichka, D. F., Tetrathiafulvalenes, Oligoacenenes, and Their Buckminsterfullerene Derivatives: The Brick and Mortar of Organic Electronics. Chem. Rev. (Washington, DC, U. S.) 2004, 104 (11), $4891-4946$.

8.Geng, Y.; Pfattner, R.; Campos, A.; Hauser, J.; Laukhin, V.; Puigdollers, J.; Veciana, J.; Mas-Torrent, M.; Rovira, C.; Decurtins, S.; Liu, S.-X., A Compact Tetrathiafulvalene-Benzothiadiazole Dyad and Its Highly Symmetrical ChargeTransfer Salt: Ordered Donor $\pi$-Stacks Closely Bound to Their Acceptors. Chem. Eur, J. 2014, 20 (23), 7136-7143.

9.Bergkamp, J. J.; Decurtins, S.; Liu, S.-X., Current advances in fused tetrathiafulvalene donor-acceptor systems. Chem. Soc. Rev. 2015, 44 (4), 863-874.

10. Souto, M.; Cui, H.; Peña-Álvarez, M.; Baonza, V. G.; Jeschke, H. O.; Tomic, M.; Valentí, R.; Blasi, D.; Ratera, I.; Rovira, C.; Veciana, J., Pressure-Induced Conductivity in a Neutral Nonplanar Spin-Localized Radical. J. Am. Chem. Soc. 2016, 138 (36), 11517-11525.

11. The original crystal structures 1 and 2, reported in previous experimental work, were uploaded and added to the Cambridge Crystallographic Data Centre (CCDC) with the reference numbers 1409550 and 1409552, respectively.

12. Cruz-Cabeza, A. J.; Reutzel-Edens, S. M.; Bernstein, J., Facts and fictions about polymorphism. Chem. Soc. Rev. 2015, 44 (23), 8619-8635.

13. Gu, C.-H.; Grant, D. J. W., Estimating the relative stability of polymorphs and hydrates from heats of solution and solubility data. J. Pharm. Sci. 2001, 90 (9), 1277-1287.

14. Demiralp, E.; Goddard, W. A., Structures and Energetics Study of Tetrathiafulvalene-Based Donors of Organic Superconductors. J. Phys. Chem. A 1997, 101 (43), 8128-8131.

15. Mukherjee, V.; Singh, N. P., Theoretical vibrational spectra and thermodynamics of organic semiconductive tetrathiafulvalene and its cation radical. Spectrochimica Acta Part A: Molecular and Biomolecular Spectroscopy 2014, 117, 315-322.

16. Giannozzi, P.; Baroni, S.; Bonini, N.; Calandra, M.; Car, R.; Cavazzoni, C.; Ceresoli, D.; Chiarotti, G. L.; Cococcioni, M.; Dabo, I.; Corso, A. D.; Gironcoli, S. d.; Fabris, S.; Fratesi, G.; Gebauer, R.; Gerstmann, U.; Gougoussis, C.; Kokalj, A.; Lazzeri, M.; Martin-Samos, L.; Marzari, N.; Mauri, F.; Mazzarello, R.; Paolini, S.; Pasquarello, A.; Paulatto, L.; Sbraccia, 
C.; Scandolo, S.; Sclauzero, G.; Seitsonen, A. P.; Smogunov, A.; Umari, P.; Wentzcovitch, R. M., QUANTUM ESPRESSO: a modular and open-source software project for quantum simulations of materials. J. Phys.: Condens. Matter 2009, 21 (39), 395502.

17. Grimme, S., Semiempirical GGA-type density functional constructed with a long-range dispersion correction. J. Comput. Chem. 2006, 27 (15), 1787-1799.

18. Rodriguez-Lopez, M. T.; Nesterov, V. N.; Youngblood, W. J., Polymorphism and DFT calculations of 1,4bis(triisopropylsilyl)buta-1,3-diyne. J. Mol. Struct. 2016, 1107, 316-321.

19. Nagendran, S.; Periyasamy, G.; Kamath, P. V., DFT Study of Polymorphism in Al(OH)3: A Structural Synthon Approach. Z. Anorg. Allg. Chem. 2015, 641 (14), 2396-2403.

20. Wen, S.; Beran, G. J. O., Crystal Polymorphism in Oxalyl Dihydrazide: Is Empirical DFT-D Accurate Enough? J. Chem. Theory Comput. 2012, 8 (8), 2698-2705.

21. Pedone, A.; Presti, D.; Menziani, M. C., On the ability of periodic dispersion-corrected DFT calculations to predict molecular crystal polymorphism in para-diiodobenzene. Chem. Phys. Lett. 2012, 541, 12-15.

22. Vela, S.; Fumanal, M.; Ribas-Arino, J.; Robert, V., Towards an accurate and computationally-efficient modelling of Fe(II)-based spin crossover materials. Phys. Chem. Chem. Phys. 2015, 17, 16306-16314.

23. Bucko, T.; Hafner, J.; Lebegue, S.; Angyan, J. G., Improved Description of the Structure of Molecular and Layered Crystals: Ab Initio DFT Calculations with van der Waals Corrections. J. Phys. Chem. A 2010, 114 (43), 11814-11824.

24. Vela, S.; Novoa, J. J.; Ribas-Arino, J., Insights into the crystal-packing effects on the spin crossover of [FeII(1-bpp)]2+based materials. Phys. Chem. Chem. Phys. 2014, 16 (48), 27012-27024.

25. Frisch, M. J.; Trucks, G. W.; Schlegel, H. B.; Scuseria, G. E.; Robb, M. A.; Cheeseman, J. R.; Scalmani, G.; Barone, V.; Mennucci, B.; Petersson, G. A.; Nakatsuji, H.; Caricato, M.; Li, X.; Hratchian, H. P.; Izmaylov, A. F.; Bloino, J.; Zheng, G.; Sonnenberg, J. L.; Hada, M.; Ehara, M.; Toyota, K.; Fukuda, R.; Hasegawa, J.; Ishida, M.; Nakajima, T.; Honda, Y.; Kitao, O.; Nakai, H.; Vreven, T.; Montgomery Jr., J. A.; Peralta, J. E.; Ogliaro, F.; Bearpark, M.; Heyd, J. J.; Brothers, E.; Kudin, K. N.; Staroverov, V. N.; Kobayashi, R.; Normand, J.; Raghavachari, K.; Rendell, A.; Burant, J. C.; Iyengar, S. S.; Tomasi, J.; Cossi, M.; Rega, N.; Millam, J. M.; Klene, M.; Knox, J. E.; Cross, J. B.; Bakken, V.; Adamo, C.; Jaramillo, J.; Gomperts, R.; Stratmann, R. E.; Yazyev, O.; Austin, A. J.; Cammi, R.; Pomelli, C.; Ochterski, J. W.; Martin, R. L.; Morokuma, K.; Zakrzewski, V. G.; Voth, G. A.; Salvador, P.; Dannenberg, J. J.; Dapprich, S.; Daniels, A. D.; Farkas, Ö.; Foresman, J. B.; Ortiz, J. V.; Cioslowski, J.; Fox, D. J. In Gaussian 09, Revision D.01, CT., G. I. W., Ed. 2009.

26. Souto, M.; Lloveras, V.; Vela, S.; Fumanal, M.; Ratera, I.; Veciana, J., Three Redox States of a Diradical AcceptorDonor-Acceptor Triad: Gating the Magnetic Coupling and the Electron Delocalization. The Journal of Physical Chemistry Letters 2016, 2234-2239. 
Table of Contents Graphic

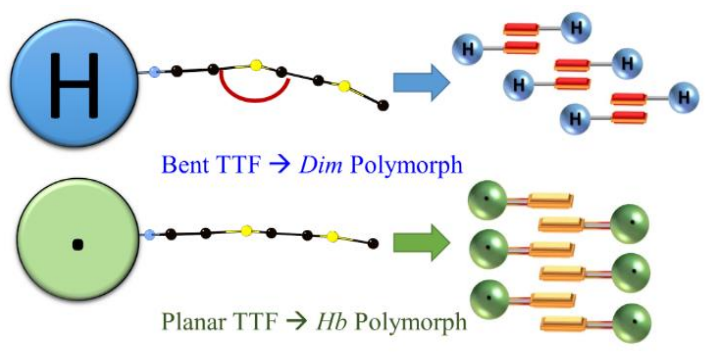

